\title{
Endodontic Management of a Tooth with Radix Entomolaris and Five Root Canals
}

\author{
Akilan Balasubramanian ${ }^{1}$, Swati Bhosale ${ }^{2}$, Maroli Ramesh Kumar ${ }^{3}$, Jayasree $\mathrm{S}^{4}$ \\ 1. (Junior Resident, Department of Conservative Dentistry and Endodontics, Government Dental College \\ Kozhikode, South India). \\ 2. (Junior Resident, Department of Conservative Dentistry and Endodontics, Government Dental College \\ Kozhikode, South India). \\ 3. (Professor and Head of the Department, Department of Conservative Dentistry and Endodontics, Government \\ Dental College Kozhikode, South India). \\ 4. (Associate Professor, Department of Conservative Dentistry and Endodontics, Government Dental College
} Kozhikode, South India).

\begin{abstract}
Success of endodontic treatment depends on the proper identification of all the canals, thorough chemo-mechanical preparation followed by three dimensional obturation with hermetic seal. Failure of any of these steps may occur due to unusual tooth morphology. The endodontic treatment of a mandibular molar with aberrant canal configuration can be diagnostically and technically challenging. Radix Entomolaris (RE) is one such aberration where an extra root is present on the distolingual aspect of mandibular first molar (molar with 3 roots). This article presents a case report of mandibular first molar with extra distal root and five root canals. Keywords: Disto lingual root, Mandibular first molar, Middle mesial canal, Radix Entomolaris.
\end{abstract}

\section{Introduction}

Knowledge of internal dental morphology is an extremely important step in planning and administering endodontic therapy. The numerous anatomical variations existing in the root canal system may contribute to the failure of root canal therapy (1). Knowledge of the most common anatomical characteristics and their possible variations is fundamental because nontreatment of even one canal can lead to endodontic treatment failure (2). The mandibular first molar which is the earliest permanent posterior tooth to erupt, seems to be the tooth that most often requires root canal treatment. Anatomical characteristics of permanent mandibular molars are generally described as a group of teeth with two roots. The usual canal distribution is two canals in the mesial root and one or two in the distal root.

In 1974, Vertucci and Wiiliams as well as Barker et al. described the presence of a middle mesial canal(3). Since then, several case reports of multiple canal systems in mandibular first molars have been investigated and described (3-5). It has been postulated that secondary dentin apposition during tooth maturation would form dentinal vertical partitions inside the root canal cavity, thus creating root canals. A third root canal may also be created inside the root canal cavity of mandibular molars by this process. Such third canals are situated centrally between the two main root canals, the buccal and lingual root canals. The diameter of those third middle canals is smaller than that of the other two (6). The probability of a mandibular first molar having a fifth canal is $1-15 \%(5)$.

The majority of first and second mandibular molars are two rooted with two mesial and one distal canal $(7,8)$. A major variant in this group is the mandibular first molar which has three roots. This variation in the number of roots is called Radix Entomolaris (RE), first mentioned in the literature by Carabelli. This has a frequency of less than $5 \%$ in white Caucasian (UK, Dutch, Finnish, German), African (Bantu Bushmen), Eurasian and Indian populations. In those with Mongoloid traits, such as the Chinese, Eskimos, and native American populations, it occurs with a frequency of 5 to more than $30 \%(9-11)$. RE has an occurrence of less than $5 \%$ in the Indian population, and such cases are rarely observed during routine endodontic procedures.

This case report presents the treatment of a Radix Entomolaris in mandibular first molar with five root canals, of which three were located in the mesial root.

\section{Case report}

A 16 year-old male patient presented with a complaint of pain in the posterior left mandibular region for the past two weeks. He gave a history of intermittent pain in the same region for the past three months. His past medical history was found to be non-contributory. Clinical examination revealed a carious left mandibular first molar. The clinical and radiographic findings led to a diagnosis of chronic irreversible pupitis of the left mandibular first molar, necessitating endodontic therapy. 
Radiographic evaluation of the involved tooth revealed normal configuration of single mesial root with two mesial canals and also the presence of a supernumerary root in addition to a mesial and a distal root. The extra root originated from the distolingual part of the tooth and appeared to be relatively straight (fig A \& B). The left inferior alveolar nerve was anesthetized using $2 \%$ Lignocaine with 1:80,000 adrenaline (Lignox, Indoco Remedies Ltd, India). The tooth was isolated using a rubber dam and an endodontic access cavity was established. Examining the fissure connecting the two mesial canals revealed an additional orifice in between the two mesial canals (mesiobuccal, middle mesial, and mesiolingual). Canal exploration with a No, $10 \mathrm{~K}$-file (Mani, Inc; Tochigi, Japan) disclosed that the distal canal was extraordinarily buccally placed, indicating some sort of variation from the normal pattern. On further exploration, the second distal canal was identified. Careful examination of access cavity revealed five distinct orifices (fig C) three located mesially (mesiobuccal, middle mesial and mesiolingual) and two distally (distobuccal and distolingual).

Multiple, working-length radiographs taken at different angulations with one file placed in each of the three mesial and two distal orifices revealed the presence of five distinct canals. The working length radiograph confirmed the presence of 5 distinct orifices and 4 apical terminations. (Fig D). Cleaning and shaping was performed using a crown down preparation with hero shaper files (Micro mega, France) nickel-titanium rotary instruments (Maillefer, Dentsply, Ballaigues, Switzerland) under abundant irrigation with $5.25 \%$ sodium hypochlorite solution and EDTA (Glyde, Maillefer, Dentsply, Ballaigues, Switzerland) in a $5 \mathrm{~mL}$ syringe. The root canals were dried with paper points (Maillefer, Dentsply, Ballaigues, Switzerland) master cone radiograph was taken (Fig E) and obturated with cold, laterally condensed gutta-percha (Maillefer, Dentsply, Ballaigues, Switzerland) and zinc oxide eugenol sealer (Dental products of India Ltd). Postobturation radiograph (Fig F) reveals 3 mesial canals where the middle mesial canal is joined with mesiobuccal canal (confluent), (additional types 3-2) and 2 distal canals (vertucci type 1).

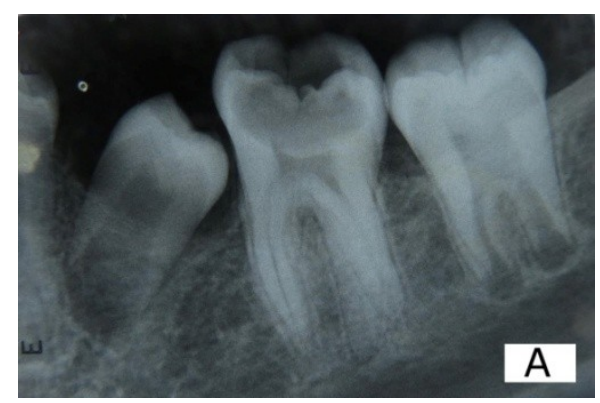

Figure A - Preoperative radiograph With $20^{\circ}$ mesial angulation showed

Extra distal root.

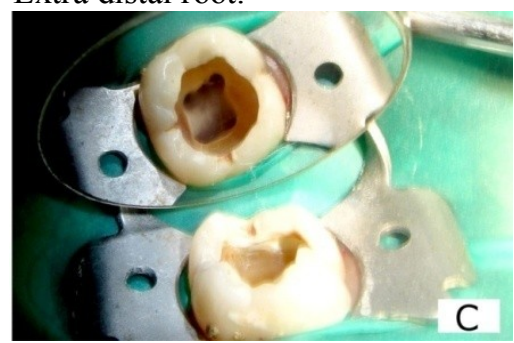

Figure C - Access cavity revealed presence of five distinct canal orifices.

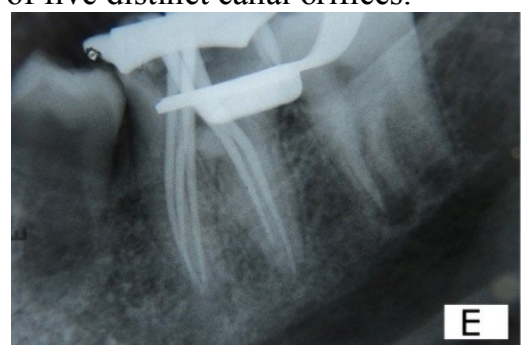

Figure E - Master cone radiograph.

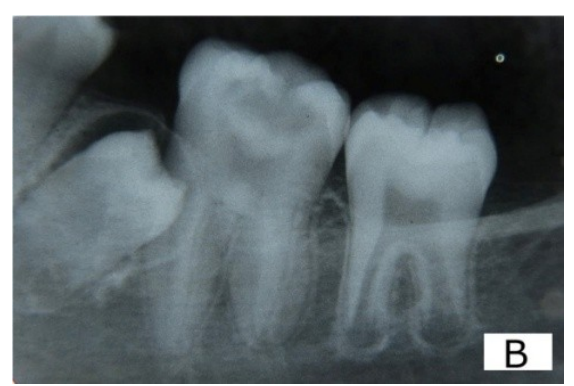

Figure B - Preoperative radiograph with $20^{0}$ distal angulation.

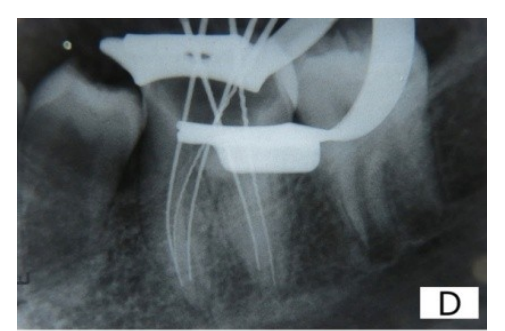

Figure D - Working length radiograph showed confluent type of middle mesial canal.

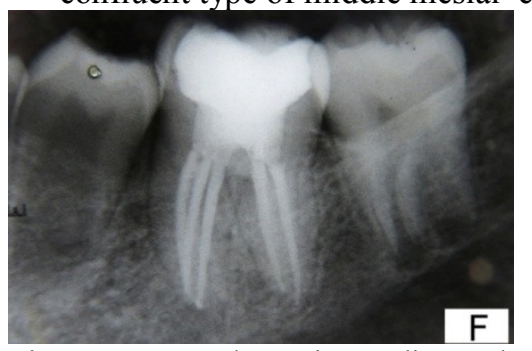

Figure F- Post obturation radiograph showing obturated mandibular first molar with radix entomolaris and middle mesial canal. 


\section{Discussion}

A thorough knowledge of root canal morphology and the configuration of the teeth plays an important role in the success of endodontic therapy (12). Several reports have described the presence of aberrant canals in the mandibular first molar that includes the presence of three canals in the mesial root $(3-5,13)$. The third mesial canal is defined as being independent when a distinct coronal orifice and apical foramen are observed, or as confluent when converging into one of the other two main canals and terminating at a common apical foramen (4). In a study of 760 mandibular molars, Fabra et al (14) found that 20 molars (2.6\%) had three canals in the mesial root. In $13(1.7 \%)$ of those, the third canal joined the mesiobuccal canal in the apical third of the root and in $6(1.6 \%)$ molars, the canals converged with the mesiolingual canal, also in the apical third. Goel et al (15) reported that the mesial root of permanent mandibular first molars presented two foramina in $60 \%$ of the specimens, whereas 6.7 and $3.3 \%$ of these molars had three and four foramina respectively.

Although a majority of the mandibular molars are two rooted with a mesial and distal root, an extra disto-lingual root may occasionally be encountered. The variation in the number of roots is called Radix Entomolaris (RE), Some authors consider a RE as a genetic trait rather than a developmental anomaly $(9,16)$. The aetiology behind the formation of RE is still unclear. Its formation could be related to: External factors during odontogenesis, Atavistic gene penetrance (Atavism is the reappearance of a trait after several generations of absence). It may be a normal morphological variant in ethnic groups of mongoloid origin $(>30 \%)$, it has rather low prevalence $(<5 \%)$ in other people such as the Indian population. $\mathrm{RE}$ is commonly found distolingually and ranges from being a short conical extension to a full-length root. The root may extend unilaterally or bilaterally (17) and may contain pulpal tissue even if it is short and conical in form(18) .

RE can be classified into four different types depending on the location of its cervical part (19).

(i)Type A: the RE is located lingually to the distal root complex which has two cone-shaped macrostructures.

(ii)Type B: the RE is located lingually to the distal root complex which has one cone-shaped macrostructures.

(iii)Type C: the RE is located lingually to the mesial root complex.

(iv)Type D: the RE is located lingually between the mesial and distal root complexes.

Each type has a sub classification to allow for the identification of separate or no separate RE.

An alternative classification of RE by De Moor et al describes the curvature of the root or the root canal (20).

(i)Type 1: a straight root or root canal.

(ii)Type 2: a curved coronal third which becomes straighter in the middle and apical third.

(iii)Type 3: an initial curve in the coronal third with a second buccally oriented curve which begins in the middle or apical third.

Endodontic success in teeth with the aforementioned number of canals and additional root requires a careful clinical and radiographic inspection(21). An additional exposure of the concerned tooth from different horizontal projections, the standard buccal-to-lingual projection, 20 degrees from the mesial and 20 degrees from the distal reveals all the basic information regarding the anatomy of the tooth (22). A significant constraint in conventional radiography is that it produces a $2 \mathrm{D}$ image of a $3 \mathrm{D}$ object, resulting in the superimposition of the overlying structures. Therefore, these radiographs are of limited value in cases with complex root canal anatomy (23). Interpretation and appraisal based on a 2D radiograph may alert the clinician to the presence of aberrant anatomy but would not be able to present the variable morphological structure of root canals and their interrelations (24). Hence, it is mandatory to use all the available diagnostic aids to locate and treat the entire root canal system (25). Nance et al (26) showed that tuned aperture, computerized tomography imaging enabled a significant increase in canal detection as compared to conventional radiography. Gopikrishna et al (24) used spiral computerized tomography for the confirmatory diagnosis of a morphological aberration in the maxillary first molar.

Once a diagnosis of additional root is reached and an access cavity has to be prepared, care should be taken to establish a "straight-line" access. With the disto-lingually located orifice of the RE a modification of the classical triangular access cavity to a trapezoidal form is required to locate and access the root canal. The laws of orifice location (27) may aid in the location of extra orifices. However, care must be taken to avoid gouging or excessive removal of dentin as this may weaken the tooth structure. Based on the literature, the majority of radices entomolaris are curved. In some cases there is an additional curve starting from the middle of the root or in the apical third. Hence using precurved files, to establish a smooth glide path to the apical segment and Nickel-Titanium rotary files for cleaning and shaping, is the desired option(28). Adequate coronal enlargement avoids hindrances in the coronal segment of the canals and easy passage of the endodontic file to the apical segment. It would also allow root canal irrigants to pass on to the apical segment in larger volumes.

Diagnostic measures such as multiple, preoperative radiographs, examination of the pulp chamber floor with a sharp explorer, troughing of the grooves with ultrasonic tips, staining the chamber floor with $1 \%$ methylene blue dye, performing the sodium hypochlorite "champagne bubble test," and visualizing canal bleeding points are all important aids in locating root canal orifices (25). A DG 16 endodontic explorer used as a pathfinder determines the angle at which the canals depart from the main chamber. The search for an extra orifice is also aided by the 
use of magnifying loupes and fibre-optic transillumination to locate the developmental line between the mesiobuccal and mesiolingual orifices. The detection of root canal orifices may be influenced by an anatomical configuration of the root canal system. Also, a better understanding of the root canal anatomy would make it easier to locate canal orifices under magnification, even if the canal anatomy was complicated.(29)

The introduction of operating microscope has revolutionized the practice of endodontics. An advantage of using the operating microscope for conventional endodontics is the enhanced visualization of root canal intricacies, which enables the clinician to investigate the root canal system and to clean and shape it more efficiently.(30)

\section{Conclusion}

Treating extra canals may be challenging but the inability to find and properly treat root canals may cause failures. Although the incidence of root and canal variations is rare, every effort should be made to find and treat all canals for successful clinical results. The possibility of an extra root should also be considered and looked for carefully. Proper angulation and interpretation of radiographs help to identify chamber and root anatomy. In the case of an RE the conventional triangular opening cavity must be modified to a trapezoidal form in order to better locate and access the distolingually located orifice of the additional root.

\section{References:}

[1]. Favieri A, Barros FG, Campos LC. Root canal therapy of a maxillary first molar with five root canals: case report. Braz Dent J 2006;17(1):75-8 Epub 2006 May 2.

[2]. Barbizam JV, Ribeiro RG, Tanomaru Filho M. Unusual anatomy of permanent maxillary molars. J Endod 2004 Sep;30(9):668-71.

[3]. Baugh D, Wallace J. Middle mesial canal of the mandibular first molar: a case report and literature review. J Endod 2004 Mar;30(3):185-6.

[4]. Holtzmann L. Root canal treatment of a mandibular first molar with three mesial root canals. Int Endod J 1997 Nov;30(6):422-3.

[5]. Navarro LF, Luzi A, Garcia AA, Garcia AH. Third canal in the mesial root of permanent mandibular first molars: review of the literature and presentation of 3 clinical reports and 2 in vitro studies. Med Oral Patol Oral Cir Bucal 2007 Dec 1;12(8):E605-9.

[6]. Martinez-Berna A, Badanelli P. Mandibular first molars with six root canals. J Endod 1985 Aug;11(8):348-52.

[7]. Vertucci FJ. Root canal anatomy of the human permanent teeth. Oral Surg Oral Med Oral Pathol 1984 Nov;58(5):589-99.

[8]. Barker BC, Parsons KC, Mills PR, Williams GL. Anatomy of root canals. III. Permanent mandibular molars. Aust Dent J 1974 Dec;19(6):408-13.

[9]. Curzon ME, Curzon JA. Three-rooted mandibular molars in the Keewatin Eskimo. J Can Dent Assoc (Tor) 1971 Feb;37(2):71-2.

[10]. Turner CG, 2nd. Three-rooted mandibular first permanent molars and the question of American Indian origins. Am J Phys Anthropol 1971 Mar;34(2):229-41.

[11]. Yew SC, Chan K. A retrospective study of endodontically treated mandibular first molars in a Chinese population. J Endod 1993 Sep;19(9):471-3.

[12]. Adanir N. An unusual maxillary first molar with four roots and six canals: a case report. Aust Dent J 2007 Dec;52(4):333 -5.

[13]. Weine FS. Case report: three canals in the mesial root of a mandibular first molar(?). J Endod 1982 Nov;8(11):517-20.

[14]. Fabra-Campos H. Three canals in the mesial root of mandibular first permanent molars: a clinical study. Int Endod J 1989 Jan;22(1):39-43.

[15]. Goel NK, Gill KS, Taneja JR. Study of root canals configuration in mandibular first permanent molar. J Indian Soc Pedod Prev Dent 1991 Mar;8(1):12-4.

[16]. Walker RT. Root form and canal anatomy of mandibular second molars in a southern Chinese population. J Endod 1988 Jul;14(7):325-9.

[17]. Somogyi-Csizmazia W, Simons AJ. Three-rooted mandibular first permanent molars in Alberta Indian children. J Can Dent Assoc (Tor) 1971 Mar;37(3):105-6.

[18]. Reichart PA, Metah D. Three-rooted permanent mandibular first molars in the Thai. Community Dent Oral Epidemiol 1981 Aug;9(4):191-2.

[19]. Carlsen O, Alexandersen V. Radix entomolaris: identification and morphology. Scand J Dent Res 1990 Oct;98(5):363-73.

[20]. De Moor RJ, Deroose CA, Calberson FL. The radix entomolaris in mandibular first molars: an endodontic challenge. Int Endod J 2004 Nov;37(11):789-99.

[21]. de Almeida-Gomes F, de Sousa BC, dos Santos RA. Unusual anatomy of mandibular premolars. Aust Endod J 2006 Apr;32(1):435 .

[22]. Klein RM, Blake SA, Nattress BR, Hirschmann PN. Evaluation of X-ray beam angulation for successful twin canal identification in mandibular incisors. Int Endod J 1997 Jan;30(1):58-63.

[23]. Holtzman L. Root canal treatment of mandibular second premolar with four root canals: a case report. Int Endod J 1998 Sep;31(5):364-6.

[24]. Gopikrishna V, Reuben J, Kandaswamy D. Endodontic management of a maxillary first molar with two palatal roots and a single fused buccal root diagnosed with spiral computed tomography - a case report. Oral Surg Oral Med Oral Pathol Oral Radiol Endod 2008 Apr;105(4):e74-8 doi: 101016/jtripleo200711022.

[25]. Vertucci FJ. Root canal morphology and its relationship to endodontic procedures. Endodontic Topics. 2005;10(1):3-29.

[26]. Nance R, Tyndall D, Levin LG, Trope M. Identification of root canals in molars by tuned-aperture computed tomography. Int Endod J 2000 Jul;33(4):392-6.

[27]. Krasner P, Rankow HJ. Anatomy of the pulp-chamber floor. J Endod 2004 Jan;30(1):5-16.

[28]. Calberson FL, De Moor RJ, Deroose CA. The radix entomolaris and paramolaris: clinical approach in endodontics. J Endod 2007 Jan;33(1):58-63 Epub 2006 Jul 26.

[29]. Yoshioka T, Kobayashi C, Suda H. Detection rate of root canal orifices with a microscope. J Endod 2002 Jun;28(6):452-3.

[30]. Saunders WP, Saunders EM. Conventional endodontics and the operating microscope. Dent Clin North Am 1997 Jul;41(3):415-28. 